\title{
Effects of Sit-to-stand Training with Various Foot Positions Combined with Visual Feedback on Postural Alignment and Balance in Stroke Patients
}

\author{
Su-Jin $\mathrm{Kim} \cdot \mathrm{Ho}-\mathrm{Hee} \mathrm{Son}^{1 \dagger}$ \\ Department of Physical Therapy, Eson Hospital Rehabilitation \\ 'Department of Physical Therapy, College of Health Sciences, Catholic University of Pusan
}

Received: September 30, 2021 / Revised: October 5, 2021 / Accepted: October 25, 2021

(C) 2021 J Korean Soc Phys Med

\section{| Abstract |}

PURPOSE: This study compared the effects of sit-to-stand training with various foot positions combined with visual feedback on the postural alignment and balance.

METHODS: Thirty stroke patients were assigned randomly into three groups of standing with a symmetrical foot position (SSF) $(n=10)$, asymmetrical foot position with the affected foot at the rear (SAF) $(n=10)$, and visual feedback and asymmetrical foot position (SVAF) $(\mathrm{n}=10)$.

Sit-to-stand training with different foot positions was performed for 30 minutes a day, five times a week, for a total of four weeks, and the effects on postural alignment and balance were assessed.

RESULTS: The angle between the midline and scapula peak of the affected side was decreased significantly at sitting and thigh-off in the SAF group and at sitting, thigh-off, and standing in the SVAF group $(\mathrm{p}<.05)$. The angle between the

$\dagger$ Corresponding Author: Ho-hee Son sonhh@cup.ac.kr, https://orcid.org/0000-0003-0905-6484 This is an Open Access article distributed under the terms of the Creative Commons Attribution Non-Commercial License (http://creativecommons.org/licenses/by-nc/3.0) which permits unrestricted non-commercial use, distribution, and reproduction in any medium, provided the original work is properly cited. midline and scapula peak of the non-affected side was increased significantly at sitting and thigh-off in the SAF group and at sitting, thigh-off, and standing in the SVAF group, the difference in the angle between the scapular peaks of the left and right sides was decreased significantly at sitting and thigh-off in SSF group, and at sitting in SAF group ( $\mathrm{p}<$ $.05)$. In the SVAF group, the angle at sitting, thigh-off, and standing was decreased significantly $(\mathrm{p}<.05)$. A comparison of the balance ability showed that BSS in the SVAF group was improved significantly $(\mathrm{p}<.05)$.

CONCLUSION: Based on these results, the postural alignment and balance ability were improved in stroke patients who participated in sit-to-stand with visual feedback and asymmetrical foot position training.

Key Words: Balance, Sit-to-stand training, Stroke, Postural alignment, visual feedback

\section{Introduction}

Stroke is a cerebrovascular disorder caused by insufficient blood supply to the brain due to ruptured or blocked blood vessels [1]. Stroke patients show an asymmetric posture, weight bearing, and muscle weakness due to neurological damages, which lead to limitations in posture control and 
balance ability, in addition to impaired motor ability caused by reduced functional activity [2].

The sit-to-stand activity is one of the most commonly performed functional activities. Stand-up motion belongs to motions that support the weight as a fixed segment in the lower extremities, and an effective ability to stand up is essential for erect bipedalism. In particular, stroke patients show erratic movements, are slow in controlling their postures, and have weak muscles, which lead to a tendency to fall while standing up [3]. In other words, an asymmetrical exercise pattern appears because of the predominant use of the non-affected side and compensation [4], which compensates for the limited movements and weakening of the muscles of the affected lower limb by moving the central point of the body to the non-affected side [5]. Such compensatory movement in stroke patients leads to greater difficulties in motor control because of the inefficient joint motions during posture control [5]. Therefore, it is essential to reduce the use of the non-affected side during movement for effective weight bearing.

Various studies on effective intervention programs to treat the non-use of the affected side caused by the predominant use of the non-affected side in stroke patients have been conducted. Inducing movement of the affected side during sitting and standing by changing the position of the non-affected foot is a method that is used mainly by stroke patients to increase the weight support of the affected side [6]. Roy et al. [7] reported that standing up with the affected foot behind the non-affected foot reduced the asymmetry of weight bearing on thighs and feet of both sides, while standing up with the non-affected foot on the back of the affected foot increased asymmetry.

Such intervention of sitting and standing by changing the position of the affected foot in stroke patients has been studied in various ways. Camargos et al. [8] assessed the effects of changing the foot position in chronic stroke patients through sit-to-stand activity on muscle activity and performance time, and Farqalit and Shahnawaz [9] examined the effects of changing the foot position through sit-to-stand activity on the balance and upright mobility in chronic stroke patients.

In clinical settings, visual feedback is used in various ways to improve the movement of the affected side. In particular, because stroke patients tend to place their feet in their visual field and use them in surrounding situations according to the movement of the feet as visual feedback [10], they are more dependent on visual feedback than other patients. Visual feedback helps stroke patients recover the brain function by enabling bilateral exercises through reflections in the mirror [11]. Stevens and Stoykov [12] provided visual feedback using a mirror to observe the functional recovery of the upper limb after a four-week exercise therapy, and Pellegrino et al. [13] reported positive effects after providing continuous visual feedback during sitting balance training in stroke patients. In addition, Cheng et al. [14] examined the effects of visual feedback on weight transfer in stroke patients.

Several studies proposed sit-to-stand interventions in various foot positions or with visual feedback in stroke patients. On the other hand, there are no sufficient studies that provided visual feedback and sit-to-stand exercise in various foot positions. Therefore, this study assessed the effects of sit-to-stand training with different foot positions combined with visual feedback on postural alignment and balance in stroke patients, thereby providing the information necessary for standing stroke patients.

\section{Methods}

\section{Subjects}

Patients diagnosed with stroke and hospitalized in a long-term care facility in South Korea were enrolled in the study. After a two-week preliminary experiment, this study was conducted on 30 subjects, and those undergoing neurodevelopmental therapy, functional rehabilitation 


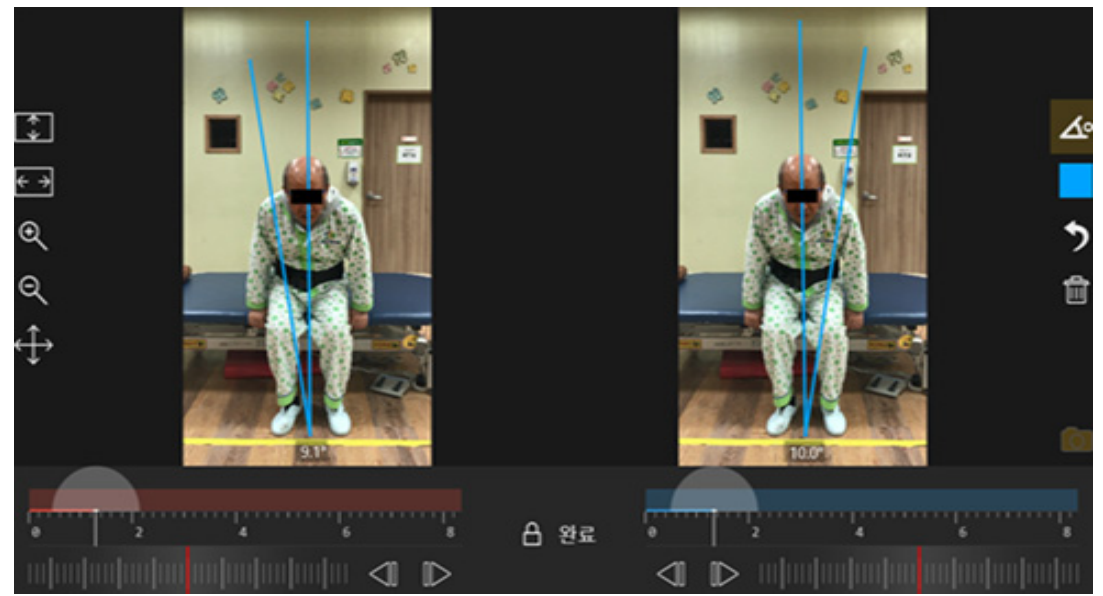

Fig. 1. myDartfish Express PC version 10.0 .

training, and functional electrical stimulation therapy. The subjects understood the purpose of the study, and agreed to participate voluntarily. The Institutional Review Board of the Catholic University of Pusan approved this study (CUPIRB-2018-030).

The selection criteria of subjects in this study were as follows:

1) Patients diagnosed with stroke more than six months earlier;

2) Patients with a score of 18 or higher in the Korean version of the mini-mental state examination and could understand tasks and communicate;

3) Those who could stand up and walk with walking aids.

The exclusion criteria were as follows.

1) Those with a history of orthopedic diseases that may affect the study;

2) Those with damages to the cerebellum and visual field;

3) Those whose heels do not touch the floor when sitting with the contracture of the plantar flexor on the affected side;

4) Those who had unilateral neglect.

\section{Measurement Method}

1) Postural Alignment

myDartfish Express PC version 10.0 (Dartfish, Fribourg, Switzerland), which was developed in Switzerland for rehabilitation and to analyze and interpret motion by recording videos with a camera, was used for analysis [15].

During the measurement, the feet were placed left and right $10 \mathrm{~cm}$ from the center of the body to maintain the same sitting posture. A camera was placed $3 \mathrm{~m}$ away from the sitting position, and markers, $2.5 \mathrm{~cm}$ in diameter, were attached to the center of both ankles and navel area. An imaginary vertical line passing through the center of the marker attached between the two ankles was drawn and set as the midline, and the angle was measured using myDartfish. The first criterion was the angle from the midline to the scapular peak on the affected and non-affected sides, as described by Shin and Lee [10]. The second criterion was the angle between the scapular peaks of the left and right sides, as described by Adamar et al. [16]. Carr and Shepherd [3] divided the sit-to-stand activity into sitting (pre-extension phase), thigh-off, and standing (extension phase), and measurements were made at each point (Fig. 1). 

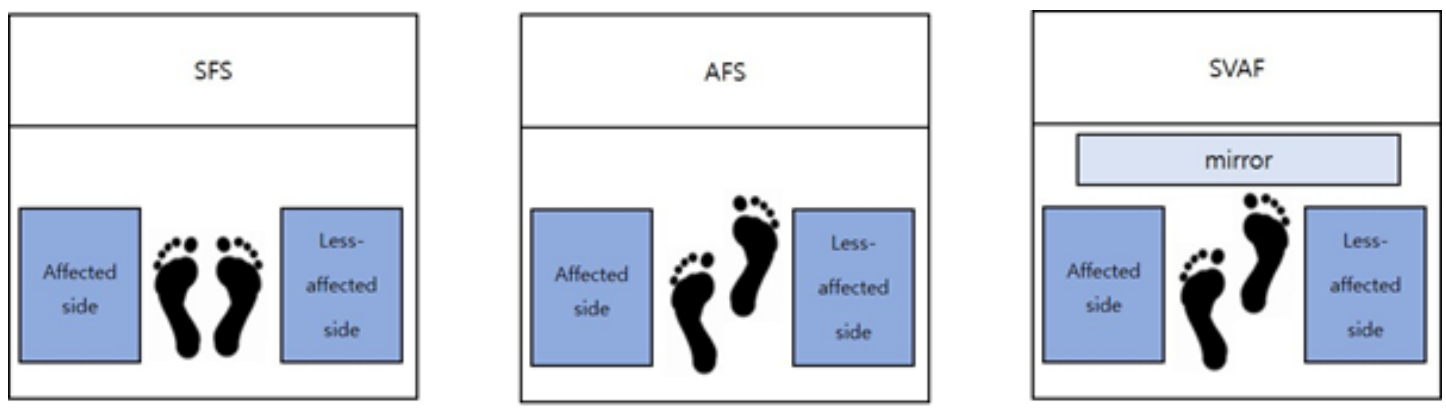

SSF: Sit to stand with symmetrical foot position

SAF: Sit to stand with asymmetrical foot position

SVAF: Sit to stand with visual feedback and asymmetrical foot position

Fig. 2. Intervention methods.

\section{2) Balance ability}

The Berg balance scale (BBS) is used widely to measure the balance ability of stroke patients. The scale consists of 14 items with a total score of 56 points on sitting in an upright position without leaning on the back of a chair, sitting in a standing position, and standing without support with both eyes closed. A lower score indicated a lower balance ability of the subject [17].

\section{Intervention Method}

Thirty patients who could understand and follow the instructions of a therapist and agreed to participate in this study were assigned randomly to three groups to perform sit-to-stand training. Six specialized physical therapists in neurodevelopmental therapy with more than five years of experience trained the subjects for 30 minutes a day, five times a week, for four weeks(Ed note: Numerals 1 to 9 are written in words.). In addition to a daily rehabilitation training program, the subjects were divided into three groups and trained to sit and stand up for 30 minutes a day in three different foot positions, including symmetrical foot position, asymmetric foot position, and asymmetric foot position with visual feedback. The subjects were rested after training (Fig. 2).
1) Sit-to-stand training with symmetrical foot position (SSF)

The chair height was adjusted to the knee height of the subject (distance between the greater trochanter and the lateral knee joint line) such that approximately half of the thighs were in contact with the chair. In a sitting position, both feet were dorsiflexed at $15^{\circ}$ and placed backward. At this time, the subjects were asked to gaze at the front. The patient stood up at a comfortable pace with both feet 15-20 $\mathrm{cm}$ apart [6].

2) Sit-to-stand training with asymmetrical foot position (SAF)

In the same posture, the foot on the affected side was dorsiflexed at $15^{\circ}$ and placed backward at a distance corresponding to $50 \%$ of the subject's foot length [6].

3) Sit-to-stand training with visual feedback and asymmetrical foot position (SVAF)

In the same posture, the foot on the affected side was dorsiflexed at $15^{\circ}$ and was placed backward at a distance corresponding to $50 \%$ of the subject's foot length from the foot on the non-affected side, and sit-to-stand training was performed in front of a full-length of mirror [6]. The subjects stood up at a comfortable pace $[6,18]$. 
Table 1. General Characteristics of Subjects $(\mathrm{N}=30)$

\begin{tabular}{cccccc}
\hline Variables & SSF $(\mathrm{n}=10)$ & SAF $(\mathrm{n}=10)$ & SVAF $(\mathrm{n}=10)$ & $\mathrm{F}$ or $\mathrm{X}^{2}$ & $\mathrm{p}$ \\
\hline Sex (Male/Female) & $5 / 5$ & $5 / 5$ & $3 / 7$ & .501 & .601 \\
Type of stroke (Inf./Hemo.) & $8 / 2$ & $7 / 3$ & $7 / 3$ & .158 & .852 \\
Onset (months) & $19.90 \pm 9.00$ & $20.30 \pm 9.12$ & $20.70 \pm 8.64$ & .186 & .980 \\
Paretic side (Lt./Rt.) & $5 / 5$ & $8 / 2$ & $5 / 5$ & 1.228 & .307 \\
Age (years) & $69.50 \pm 11.25$ & $74.10 \pm 6.77$ & $73.50 \pm 8.04$ & .718 & .508 \\
Height (cm) & $160.67 \pm 7.01$ & $161.46 \pm 7.24$ & $162.11 \pm 3.37$ & .123 & .889 \\
Body weight (kg) & $60.09 \pm 12.94$ & $60.43 \pm 9.33$ & $64.06 \pm 9.81$ & .375 & .695 \\
MMSE-K & $19.40 \pm 5.27$ & $19.70 \pm 5.45$ & $21.40 \pm 4.54$ & .404 & .672 \\
\hline
\end{tabular}

Mean \pm SD

Inf. : Infarction / Hemo. : Hemorrhage

MMSE-K: Mini Mental Scale Examination-Korean

SSF: Sit to stand with symmetrical foot position

SAF: Sit to stand with asymmetrical foot position

SVAF: Sit to stand with visual feedback and asymmetrical foot position

\section{Data Analysis}

IBM SPSS Statistics 24.0 program was used for statistical analysis. Repeated measures ANOVA were performed to compare the changes at different points of standing in postural alignment analysis. A paired t-test was used to compare the groups' differences between the pre-test and post-test values. The statistical significance level (a) was set to .05 .

\section{Results}

\section{General Characteristics of Subjects}

The subjects of this study were 30 stroke patients, and the general characteristics of the subjects were as follows (Table 1).

\section{Postural Alignment at Different Points of Sit-to-stand Training}

A. Angle between the midline and the scapula peak of the affected side

The angle between the midline and scapula peak of the affected side was measured at different points of sit-to-stand activity. In the SAF group, the angles at sitting and thigh-off decreased significantly from $16.05 \pm 2.78^{\circ}$ to $15.51 \pm .88^{\circ}$ and from $15.43 \pm 3.44^{\circ}$ to $14.62 \pm 1.07^{\circ}$, respectively, indicating that asymmetry was improved $(p<.05)$. In the SVAF group, the angles at sitting, thigh-off, and standing were significantly decreased from $14.98 \pm 2.17^{\circ}$ to 13.20 $\pm .73^{\circ}$, from $16.98 \pm 2.02^{\circ}$ to $14.42 \pm .60^{\circ}$, and from 16.53 $\pm 3.85^{\circ}$ to $14.26 \pm 1.09^{\circ}$, respectively, indicating that the asymmetry was improved $(\mathrm{p}<.05)$. In the SSF group, there was a significant difference at different stages before and after intervention $(\mathrm{p}<.05)$, and there were no significant differences within the groups $(\mathrm{p}>.05)$. (Table 2$)$.

B. Angle between the midline and the scapula peak of the non-affected side

The angle between the midline and scapula peak of the non-affected side was measured at different stages of sit-to-stand activity. In the SAF group, the angles at sitting and thigh-off increased significantly from $14.71 \pm 3.23^{\circ}$ to $15.86 \pm 3.02^{\circ}$ and from $15.51 \pm 4.29^{\circ}$ to $16.79 \pm 4.17^{\circ}$, respectively, indicating that asymmetry was improved ( $\mathrm{p}$ $<.05$ ). In the SVAF group, the angles at sitting, thigh-off, and standing increased significantly from $13.15 \pm 1.91^{\circ}$ to $15.92 \pm 2.44^{\circ}$, from $15.86 \pm 2.35^{\circ}$ to $18.76 \pm 2.06^{\circ}$, and 
Table 2. Angle of the Midline to Scapular Peak on the Affected Side at Different Stages of Sit-to-stand Activity

\begin{tabular}{|c|c|c|c|c|c|c|}
\hline & & Sitting & Thigh off & Standing & $F$ & $\mathrm{p}$ \\
\hline \multirow{4}{*}{$\begin{array}{c}\text { SSF } \\
(n=10)\end{array}$} & pre & $13.05 \pm 2.95$ & $14.83 \pm 1.97$ & $16.88 \pm 2.33$ & 6.110 & $.000^{*}$ \\
\hline & post & $12.82 \pm .93$ & $14.02 \pm 2.35$ & $16.58 \pm 2.49$ & 5.395 & $.001^{*}$ \\
\hline & $\mathrm{t}$ & 1.914 & 2.596 & 2.229 & & \\
\hline & $\mathrm{p}$ & .085 & .123 & .063 & & \\
\hline \multirow{4}{*}{$\begin{array}{c}\text { SAF } \\
(n=10)\end{array}$} & pre & $16.05 \pm 2.78$ & $15.43 \pm 3.44$ & $13.89 \pm 3.41$ & 1.185 & .324 \\
\hline & post & $15.51 \pm .88$ & $14.62 \pm 1.07$ & $13.72 \pm .78$ & .973 & .400 \\
\hline & $\mathrm{t}$ & 4.095 & 3.678 & .293 & & \\
\hline & $\mathrm{p}$ & $.000 *$ & $.001 *$ & .775 & & \\
\hline \multirow{4}{*}{$\begin{array}{c}\text { SVAF } \\
(\mathrm{n}=10)\end{array}$} & pre & $14.98 \pm 2.17$ & $16.98 \pm 2.02$ & $16.53 \pm 3.85$ & 1.393 & .265 \\
\hline & post & $13.20 \pm .73$ & $14.42 \pm .60$ & $14.26 \pm 1.09$ & .627 & .542 \\
\hline & $\mathrm{t}$ & 3.608 & 3.537 & 4.538 & & \\
\hline & $\mathrm{p}$ & $.002^{*}$ & $.000^{*}$ & $.005^{*}$ & & \\
\hline
\end{tabular}

unit; degree

Mean \pm SD

SSF: Sit to stand with symmetrical foot position

SAF: Sit to stand with asymmetrical foot position

SVAF: Sit to stand with visual feedback and asymmetrical foot position

${ }^{*}$ : significant difference $(\mathrm{p}<.05)$

Table 3. Angle of the Midline to Scapular Peak on the Non-affected Side at Different Stages of the Sit-to-stand Activity

$(\mathrm{N}=30)$

\begin{tabular}{|c|c|c|c|c|c|c|}
\hline & & Sitting & Thigh off & Standing & $\mathrm{F}$ & $\mathrm{p}$ \\
\hline \multirow{4}{*}{$\begin{array}{c}\text { SSF } \\
(n=10)\end{array}$} & pre & $14.83 \pm 3.48$ & $17.35 \pm 2.83$ & $17.19 \pm 4.51$ & 1.475 & .242 \\
\hline & post & $14.51 \pm 3.23$ & $18.31 \pm 3.35$ & $17.57 \pm 4.69$ & 2.787 & .085 \\
\hline & $\mathrm{t}$ & .848 & -2.063 & -1.764 & & \\
\hline & $\mathrm{p}$ & .425 & .064 & .117 & & \\
\hline \multirow{4}{*}{$\begin{array}{c}\text { SAF } \\
(n=10)\end{array}$} & pre & $14.71 \pm 3.23$ & $15.51 \pm 4.29$ & $15.93 \pm 4.23$ & .247 & .783 \\
\hline & post & $15.86 \pm 3.02$ & $16.79 \pm 4.17$ & $16.52 \pm 3.56$ & .172 & .847 \\
\hline & $\mathrm{t}$ & -6.535 & -4.657 & -1.377 & & \\
\hline & $\mathrm{p}$ & $.002 *$ & $.000^{*}$ & .202 & & \\
\hline \multirow{4}{*}{$\begin{array}{c}\text { SVAF } \\
(\mathrm{n}=10)\end{array}$} & pre & $13.15 \pm 1.91$ & $15.86 \pm 2.35$ & $15.05 \pm 4.01$ & 2.292 & .123 \\
\hline & post & $15.92 \pm 2.44$ & $18.76 \pm 2.06$ & $17.72 \pm 3.46$ & 2.783 & .801 \\
\hline & $\mathrm{t}$ & -5.764 & -4.406 & -4.984 & & \\
\hline & $\mathrm{p}$ & $.001^{*}$ & $.003^{*}$ & $.000^{*}$ & & \\
\hline
\end{tabular}

unit; degree

Mean \pm SD

SSF: Sit to stand with symmetrical foot position

SAF: Sit to stand with asymmetrical foot position

SVAF: Sit to stand with visual feedback and asymmetrical foot position

*: significant difference $(p<.05)$

from $15.05 \pm 4.01^{\circ}$ to $17.72 \pm 3.46^{\circ}$, respectively, indicating that asymmetry was improved $(p<.05)$. There were no significant differences at different points of sit-to-stand acti vity between the groups $(p>.05)($ Table 3$)$. 
Table 4. Angle between the Scapular Peaks at Different Stages of Sit-to-stand Activity $(N=30)$

\begin{tabular}{|c|c|c|c|c|c|c|}
\hline & & Sitting & Thigh off & Standing & $\mathrm{F}$ & $\mathrm{p}$ \\
\hline \multirow{4}{*}{$\begin{array}{c}\text { SSF } \\
(\mathrm{n}=10)\end{array}$} & pre & $2.51 \pm .60$ & $4.49 \pm 2.02$ & $2.49 \pm 1.20$ & 2.068 & .091 \\
\hline & post & $2.35 \pm .59$ & $3.94 \pm 1.79$ & $2.23 \pm .90$ & .791 & .185 \\
\hline & $\mathrm{t}$ & 4.000 & 2.807 & 1.273 & & \\
\hline & $\mathrm{p}$ & $.000^{*}$ & $.002 *$ & .243 & & \\
\hline \multirow{4}{*}{$\begin{array}{c}\text { SAF } \\
(n=10)\end{array}$} & pre & $3.20 \pm 1.07$ & $5.09 \pm 2.34$ & $3.13 \pm .86$ & 2.393 & .077 \\
\hline & post & $2.85 \pm .84$ & $4.68 \pm 2.04$ & $3.11 \pm 1.63$ & .278 & .101 \\
\hline & $\mathrm{t}$ & 2.704 & .817 & .003 & & \\
\hline & $\mathrm{p}$ & $.002 *$ & .433 & .971 & & \\
\hline \multirow{4}{*}{$\begin{array}{c}\text { SVAF } \\
(\mathrm{n}=10)\end{array}$} & pre & $3.62 \pm 1.57$ & $5.06 \pm 1.86$ & $2.96 \pm 1.21$ & 1.982 & .717 \\
\hline & post & $2.80 \pm 1.42$ & $4.13 \pm 1.53$ & $2.59 \pm 1.06$ & 2.010 & .844 \\
\hline & $\mathrm{t}$ & 3.055 & 2.907 & 1.807 & & \\
\hline & $\mathrm{p}$ & $.001 *$ & $.003^{*}$ & .100 & & \\
\hline
\end{tabular}

unit; degree

Mean \pm SD

SSF: Sit to stand with symmetrical foot position

SAF: Sit to stand with asymmetrical foot position

SVAF: Sit to stand with visual feedback and asymmetrical foot position

${ }^{*}:$ significant difference $(\mathrm{p}<.05)$

C. Difference in the angle between the scapular peaks of the left and right sides

In the SSF group, the differences of the angle between the scapular peaks of the left and right sides at the sitting and thigh-off decreased significantly from $2.51 \pm .60^{\circ}$ to $2.35 \pm .59^{\circ}$ and from $4.49 \pm 2.02^{\circ}$ to $3.94 \pm 1.79^{\circ}$, respectively, indicating that asymmetry was improved (p $<.05)$. In the SAF group, the differences were decreased significantly from $3.20 \pm 1.07^{\circ}$ to $2.85 \pm .84^{\circ}$, indicating that the asymmetry had improved $(\mathrm{p}<.05)$. In the SVAF group, the differences at sitting and thigh-off decreased significantly from $3.62 \pm 1.57^{\circ}$ to $2.80 \pm 1.42^{\circ}$ and from $5.06 \pm 1.86^{\circ}$ to $4.13 \pm 1.53^{\circ}$, respectively, suggesting that asymmetry was improved $(p<.05)$. There were no significant differences at different points of sit-to-stand activity between the groups $(\mathrm{p}>.05)($ Table 4$)$.

\section{Comparison of Balance Ability}

When the BBS test was performed, the score increased from $19.90 \pm 16.06$ to $20.00 \pm 16.12$ and from $20.90 \pm$ 16.76 to $21.20 \pm 16.69$ in the SSF and SAF groups, respectively. The increase was not significant in both groups $(p>.05)$. In the SVAF group, the score increased significantly from $16.90 \pm 13.28$ to $17.70 \pm 13.72$ after the intervention, indicating that the balance ability was improved $(p<.05)$. An intra-group comparison of the preand post-intervention values did not show significant differences $(p>.05)($ Table 5$)$.

\section{Discussion}

In this study, sit-to-stand training in a symmetrical foot position, asymmetrical foot position, and asymmetrical foot position with visual feedback was performed for four weeks to assess the effects on the postural alignment and balance of stroke patients.

When the postural alignments at different points of standing were analyzed using the myDartfish software 
Table 5. Berg Balance Scale between and Within the Groups

\begin{tabular}{cccccc}
\hline & SSF $(\mathrm{n}=10)$ & SAF $(\mathrm{n}=10)$ & SVAF $(\mathrm{n}=10)$ & $\mathrm{F}$ & $\mathrm{p}$ \\
\hline pre & $19.90 \pm 16.06$ & $20.90 \pm 16.76$ & $16.90 \pm 13.28$ & .181 & .830 \\
post & $20.00 \pm 16.12$ & $21.20 \pm 16.69$ & $17.70 \pm 13.72$ & .134 & .875 \\
$\mathrm{t}$ & -1.030 & -1.401 & -2.751 & & \\
$\mathrm{p}$ & .354 & .119 & $.002^{*}$ & \\
\hline
\end{tabular}

unit; score

Mean \pm SD

SSF: Sit to stand with symmetrical foot position

SAF: Sit to stand with asymmetrical foot position

SVAF: Sit to stand with visual feedback and asymmetrical foot position

${ }^{*}$ : significant difference $(\mathrm{p}<.05)$

program, the angle between the midline and scapula peak of the affected side decreased significantly at sitting and thigh-off in the SAF group and at sitting, thigh-off, and standing in the SVAF group, indicating that asymmetry was improved $(\mathrm{p}<.05)$. The angle between the midline and scapula peak of the non-affected side increased significantly at sitting and thigh-off in the SAF group and at sitting, thigh-off, and standing in the SVAF group, indicating that asymmetry was improved $(p<.05)$. In the SSF group, the angle between the scapular peaks of the left and right sides at the sitting and thigh-off decreased significantly. In the SAF group, the angle at sitting decreased significantly. In addition, in the SVAF group, the angle at sitting, thigh-off, and standing significantly decreased, indicating that the asymmetry was improved in all three groups $(p<.05)$.

The sit-to-stand activity requires the ability to move the center of gravity within the narrowed base stably, and it is an important activity for an independent life [18]. According to Carr and Shepherd [3], sit-to-stand can be divided into sitting (pre-extension phase), thigh-off, and standing (extension phase). In the pre-extension phase, the foot was moved to place the ankle joint behind the knee joint. When the knee moves forward, the angular velocity generated by the upper body leads to ground repulsion that causes the thigh to be lifted. In the extension phase, the lower limb extensors vertically accelerate the body. During the acute stage, stroke patients tend to adapt to asymmetrical weight bearing, which places less weight on the lower extremity of the affected side, in which standing is difficult to perform [20]. Canning et al. [21] reported that an increased weight load on the lower extremity of the affected side is effective for function recovery as asymmetrical weight bearing during standing in stroke patients is the leading cause of falls.

Ehrsson et al. [22] suggested that visual feedback using a mirror is a cognitive technique that can help increase the motor function of stroke patients. In particular, interventions using mirrors, which provide visual feedback as if the movement of the non-affected limb reflected in the mirror is the movement of the affected limb and promote function [13], are effective in stroke rehabilitation. In the present study, visual feedback using a mirror in sit-to-stand training helped reduce asymmetry significantly at all points of standing, which was consistent with the previous study findings.

Shin and Lee [10] investigated sit-to-stand activity in 41 stroke patients and 35 healthy adults by varying the conditions of visual feedback (eye-open vs. eye-closed) and base-support (both-side support, affected-side support, and unaffected-side support). The conditions on posture control were analyzed with myDartfish. Stroke patients 
included their feet into the visual field and perceived the surrounding situation according to the movement of the feet as feedback [10]. Ju et al. [23] reported that visual cues to align the body reduce sway of the affected lower extremity, leading to no significant difference in swaying compared to the non-affected lower extremity. Therefore, visual feedback during sit-to-stand training had positive effects on controlling the postural alignment. This finding is consistent with other studies that reported that various motor therapies combined with visual feedback were effective.

Moreover, standing with symmetrical and asymmetrical positions and the asymmetric position and visual feedback also positively affected postural alignment. In other words, the sit-to-stand activity is one of the ADL in which all body segments change periodically, and symmetrical muscle performance of the left and right lower extremities and trunk muscles and weight support of both lower extremities are essential to maintain body balance [24]. Therefore, standing with symmetrical and asymmetrical foot positions without visual feedback is also considered effective interventions to control postural alignment.

The balance ability was compared using the BSS test, and it was observed that the SVAF group showed improved balance ability $(\mathrm{p}<.05)$. Ji et al. [18] examined 26 stroke patients (13 patients assigned to exercise in front of a mirror and 13 patients exercised without a mirror), and observed that the BSS score was improved significantly in the experimental group who exercised in front of a mirror, indicating that the balance was improved. In the absence of normal somatosensory sensation, visual feedback restores the flow of information from the inferior parietal lobule to the motor cortex, and the exercise program, which is reprinted from the motor cortex, promotes the movements of the limbs [25]. Lehmann et al. [26] suggested measuring the left and right swaying and performing balance training using visual feedback as a reliable and effective intervention for stroke patients. Therefore, the study findings are consistent with those of other studies showing that combining exercise therapies with visual feedback is effective.

In this study, visual feedback was combined with sit-to-stand activity with asymmetrical foot position, and video recording was acquired to analyze the alignment at different stages of sit-to-stand activity. The following are the strengths of this study. First, in many previous studies, the left, right, and anteroposterior sway distances were analyzed to assess posture control. In the present study, the angle was divided into the sagittal and coronal planes for detailed analysis. Second, although there was a large distribution of chronic stroke patients, significant functional improvements were observed after a short four-week intervention.

On the other hand, the number of subjects included in this study was small, which limits the generalization of the results to all patients with brain lesions. Therefore, systematic studies with more subjects will be necessary.

\section{Conclusion}

This study examined the effects of sit-to-stand activity in three different feet positions on postural alignment and balance of stroke patients. Thirty stroke patients were assigned to three different groups, and the pre- and postintervention results were compared and analyzed as follows.

First, the angle between the midline and scapula peak of the affected side was decreased at sitting and thigh-off in the SAF group and at sitting, thigh-off, and standing in the SVAF group, indicating that asymmetry was significantly improved $(\mathrm{p}<.05)$. The angle between the midline and scapula peak of the non-affected side was significantly increased at sitting and thigh-off in the SAF group and at sitting, thigh-off, and standing in the SVAF group, indicating that asymmetry was improved $(\mathrm{p}<.05)$. In the SSF group, the angle between the scapular peaks of the left and right sides at sitting and thigh-off was significantly decreased, and in the SAF group, the angle 
at sitting was decreased significantly. In addition, in the SVAF group, the angle at sitting, thigh-off, and standing was significantly decreased, indicating that asymmetry was improved in all three groups $(\mathrm{p}<.05)$. Second, a comparison of balance ability showed that BSS in the SVAF group was significantly improved $(\mathrm{p}<.05)$.

The SVAF group showed overall improvement in function compared to the SSF group. Therefore, training programs, including visual sensory stimuli, would be necessary for efficient postural alignment and balance improvement when developing intervention programs for stroke patients in the future.

\section{References}

[1] Sullivan KJ, Brown DA, Klassen T, et al. Effects of task-specific locomotor and strength training in adults who were ambulatory after stroke: results of the STEPS randomized clinical trial. Phys Ther. 2007;87(12):1580602.

[2] Eng JJ, Chu KS. Reliability and comparison of weightbearing ability during standing tasks for individuals with chronic stroke. Arch Phys Med Rehabil. 2002;83(8): 1138-44.

[3] Carr JH, Shepherd RB. neurological Rehabilitation 2 edition, 2010.

[4] Lee JH, Min DK, Choe HS, et al. The effects of upper and lower limb position on symmetry of vertical ground reaction force during sit-to-stand in chronic stroke subjects. J Phys Ther Sci. 2018;30(2):242-7.

[5] Thielman G, Kaminski T, Gentile AM. Rehabilitation of reaching after stroke: Comparing 2 training protocols utilizing trunk restraint. Neurorehabil Neural Repair. 2008;22(6):697-705.

[6] Gray CK, Culham E. Sit-to-Stand in People with Stroke: Effect of Lower Limb Constraint-Induced Movement. Strategies Stroke Res Treat. 2014;681-3.

[7] Roy G, nadeau S, Gravel D, et al. Side difference in the hip and knee joint moments during sit-to-stand and stand-to-sit tasks in individuals with hemiparesis. Clin Biomech (Bristol, Avon). 2007;22(7):795-804.

[8] Camargos A, Rodrigues F, Teixeira F. The effects of foot position on the performance of the sit-to-stand movement with chronic stroke subjects. Arch Phys Med Rehabil. 2009;90(2):314-9.

[9] Farqalit R, Shahnawaz A. Effect of foot position during sit-to-stand training on balance and upright mobility in patients with chronic stroke. Hong Kong Physiother J. 2013;31(2):75-80.

[10] Shin JB, Lee JS. The Effect of Visual Feedback on Postural Control During Sit-to-Stand Movements of BrainDamaged Patients Under Different Support Conditions. Phys Ther Korea, 2012;19(3):40-50.

[11] Yavuzer G, Selles R, Sezer N, et al. Mirror therapy improves hand function in subacute stroke: a randomized controlled trial. Arch Phys Med Rehabil. 2008;89(3): 393-8.

[12] Stevens JA, Stoykov ME. Using motor imagery in the rehabilitation of hemiparesis. Arch Phys Med Rehabil. 2003;84(7):1090-2.

[13] Pellegrino L, Giannoni P, Marinelli L, et al. Effects of continuous visual feedback during sitting balance training in chronic stroke survivors. J Neuroeng Rehabil. 2017; 1(1) $4: 107$.

[14] Cheng PT, Chen CL, Wang CM, et al. Leg Muscle Activation Patterns of Sit-to-Stand Movement in Stroke Patients. Am J Phys Med Rehabil. 2004;83(1):10-6.

[15] Goetz L, Piallat B, Thibaudier Y, et al. A non-human primate model of bipedal locomotion under restrained condition allowing gait studies and single unit brain recordings. J Neurosci Methods. 2012;204(2):306-17.

[16] Adamar n, Juliana M, Yeda P, et al. Head and shoulder alignment among patients with unilateral vestibular hypofunction. Rev Bras Fisioter. 2010;14(4):330-6.

[17] Berg K, Wood-Dauphinee S, Williams JI. The balance scale: Reliability assessment with elderly residents and 
patients with an acute stroke. Scand J Rehabil Med. 1995;27(1):27-36.

[18] Ji S, Nam G, Kim M, et al. The Effect of Visual Feedback Training Using a Mirror on the Balance in Hemiplegic Patients. J Korean Soc Phys Med. 2011;6(2):153-63.

[19] Demura S, Sato S, Minami M, et al. Gender and age differences in basic ADL ability on the elderly: Comparison between the independent and the dependent elderly. J Physiol Anthropol Appl Human Sci. 2003; 22(1):19-27.

[20] Britton E, Harris n, Turton A. An exploratory randomized controlled trial of assisted practice for improving sitto-stand in stroke patients in the hospital setting. Clin Rehabil. 2008;22(5):458-68.

[21] Canning CG, Shepherd RB, Carr JH, et al. A randomized controlled trial of the effects of intensive sit-to-stand training after recent traumatic brain injury on sit-to-stand performance. Clin Rehabil. 2003;17(4):355-62.
[22] Ehrsson HH, Spence C, Passingham RE. That's my hand! Activity in premotor cortex reflects feeling of ownership of a limb. Science. 2004;305(5685):875-7.

[23] Ju S, Yoo WG, Oh JS, et al. Effects of visual cue and cognitive motor tasks on standing postural control following a chronic stroke. J Phys Ther Sci. 2018;30(4): 601-4.

[24] Goulart FR, Valls-Solé. Patterned electromyographic activity in the sit-to-stand movement. Clin Neurophysiol. 1999;110(9):1634-40.

[25] Thieme H, Morkisch N, Mehrholz J, et al. Mirror therapy for improving motor function after stroke. Cochrane Database Syst Rev. 2018;7(7):CD008449. Published 2018 Jul 11.

[26] Lehmann JF, Boswell S, Price R, et al. Quantitative evaluation of sway as an indicator of functional balance in post traumatic brain injury. Arch Phys Med Rehabil. 1990;71(12):955-62. 